\title{
Echantillonnage et modélisation de la dynamique des peuplements forestiers Application au cas de l'inventaire forestier national
}

\author{
François HOULLIER
}

\begin{abstract}
L'auteur, ingénieur du Génie rural des Eaux et des Forêts, a mené à bien cette thèse simultanément auprès de la Station de Sylviculture et production du Centre de recherches forestières de Nancy-Champenoux (I.N.R.A.) et du laboratoire de biométrie de l'Université de Lyon I, dans le cadre de la formation complémentaire par la recherche mise en place par l'E.N.G.R.E.F.

Elle a été soutenue à l'Université Claude Bernard (Lyon I) le 6 juin 1986 devant un jury composé de J. Boucon, J.M. Legay, C. Millier, A. Pavé, R. Schlaepfer et J.P. TroY.
\end{abstract}

En voici le résumé :

Les inventaires forestiers de production répondent, à différentes échelles, à trois types de questions: l'estimation de la ressource en bois et de ses variations passées et la prédiction de l'évolution future des peuplements. Deux familles de méthodes mathématiques sont employées pour parvenir à ces objectifs : l'échantillonnage et la modélisation. Ce travail vise à expliciter les liens entre ces méthodes, lorsqu'on s'intéresse à l'évolution dynamique des peuplements forestiers.

La première partie est organisée autour de l'idée d'interaction entre les objectifs de l'utilisateur, les structures de la population et des données et les méthodes de traitement. On envisage d'abord une stratégie d'échantillonnage temporel, « l'échantillonnage avec remesure partielle " et sa généralisation spatio-temporelle par "la théorie des variables régionalisées ". On brosse ensuite un panorama des principes de la modélisation de la dynamique des peuplements forestiers : ces concepts généraux sont illustrés sur un matériel méthodologique particulier, les courbes de croissance. Une synthèse sur l'articulation entre échantillonnage et modélisation est enfin proposée sur la base d'un modèle probabiliste structurel de la population étudiée.

La deuxième partie est centrée sur des applications au cas de l'Inventaire Forestier National (I.F.N.), dont on présente d'abord la problématique et les données. On envisage alors la représentation de l'évolution des peuplements au moyen d'un modèle global, qui généralise les modèles démographiques (déterministes ou stochastiques) au cas des populations structurées en stades. Les limites des modèles globaux conduisent à étudier des modèles plus détaillés, relatifs au peuplement et/ou à l'arbre, et à analyser les méthodes d'ajustement de ces modèles aux données de l'I.F.N. On met alors en évidence l'imbrication des structures liées à l'arbre et au peuplement et on propose un modèle de répartition de l'accroissement en surface terrière.

Mots clés : Stratégie d'échantillonnage, modélisation, inventaire forestier, dynamique des populations, courbes de croissance. 